\title{
Short-chain chlorinated paraffins in the soils of two different Chinese cities: Occurrence, homologue patterns and vertical migration
}

Yumei Huang ${ }^{1,2}$, Laiguo Chen ${ }^{* 1,3}$, Yongbin Feng ${ }^{1,3}$, Zhixiang Ye $^{3}$, Qiusheng $\mathrm{He}^{4}$, Qianhua Feng ${ }^{1}$, Xian Qing ${ }^{1}$, Ming Liu $^{1}$, Bo Gao ${ }^{1}$

1. Urban Environment and Ecology Research Center, South China Institute of Environmental Sciences (SCIES), Ministry of Environmental Protection, Guangzhou, 510655, China

2. School of Environmental Science and Engineering, Guangzhou University, Guangzhou, 510006, China

3. College of Resource and Environment, Chengdu University of Information Technology, Chengdu, 610225, China

4. School of Environment and Safety, Taiyuan University of Science and Technology, Taiyuan, 030024, China

\begin{abstract}
Short-chain chlorinated paraffins (SCCPs) are candidate persistent organic pollutants (POPs) that are under review by the Stockholm Convention. China is currently the largest producer and consumer of chlorinated paraffins (CPs). To study the environmental behavior and fate of SCCPs in the soils of urban and suburban regions, the SCCP concentrations in 88 topsoils and 15 soil columns from land of different use types (e.g., woodland, vegetable field, paddy field and greenbelt) from Guangzhou and Chengdu have been determined. The SCCP concentrations in topsoils from Guangzhou (range: $1.45-25.5 \mathrm{ng} \mathrm{g}^{-1}$ dry weight (dw), average: $10.3 \mathrm{ng} \mathrm{g}^{-1} \mathrm{dw}$ ) were much higher than those from Chengdu (range: $0.218-3.26 \mathrm{ng} \mathrm{g}^{-1} \mathrm{dw}$, average: $1.43 \mathrm{ng} \mathrm{g}^{-1} \mathrm{dw}$ ). When compared to previously reported SCCP levels for topsoils from other areas, the SCCP concentrations measured in the present work were quite low. Much higher SCCP concentrations were observed in the greenbelt topsoils from Chengdu relative to the values measured from woodlands and vegetable and paddy fields. The composition profiles suggest that $\mathrm{C}_{10} \mathrm{Cl}_{6-10}$ and $\mathrm{C}_{11-13} \mathrm{Cl}_{6-8}$ were the major groups of SCCPs in topsoils from the woodlands and vegetable and paddy fields in Guangzhou and Chengdu. Vertical variations of the SCCP concentrations in the soil columns suggest that less chlorinated SCCPs $\left(\mathrm{Cl}_{5-6}-\mathrm{SCCPs}\right)$ are more capable of migrating to the deeper-layer soils than more chlorinated ones $\left(\mathrm{Cl}_{9-10}-\mathrm{SCCPs}\right)$. The SCCP concentrations displayed little dependence on organic matter $(\mathrm{OM})$ for most topsoils $(p>0.05)$, indicating that $\mathrm{OM}$ is not the controlling factor in the distribution of SCCPs in the soils.
\end{abstract}

Capsule: This study analyzed the occurrence, homologue patterns and vertical migration of SCCPs in the topsoils of two Chinese cities with different industrial structures and climate conditions. 
Keywords: short-chain chlorinated paraffins (SCCPs); soil; composition profile; vertical variation; organic matter

\section{Introduction}

Short-chain chlorinated paraffins (SCCPs) are chlorinated paraffins (CP) with 10-13 carbon atoms (Bayen et al., 2006). Currently, SCCPs are listed as candidate persistent organic pollutants (POPs) under review by the Stockholm Convention because of their stability, bioaccumulation, long-range transport potential and toxic character (POPRC, 2015). SCCP mixtures have been extensively used in applications including metalworking lubricants, paints, adhesives, sealants, leather fat liquors, plastics, rubber, flame retardants, textiles and polymeric materials (POPRC, 2015). Further, the increasing demand for CPs has made China the world's largest producer and user of CPs, with the total CP production reaching 1,050,300 tons in 2013 (WCC, 2014). The major CP products in China are CP-42, CP-52 and CP-70 (Sun et al., 2005; Tang and Yao, 2005), with CP-52 accounting for nearly $90 \%$ of the total 2012 output (WCC, 2014). The SCCP mass fractions in CP-42, CP-52, and CP-70 were determined to be $3.7 \%, 24.9 \%$, and $0.5 \%$, respectively (Gao et al., 2012). Large quantities of SCCPs can be released into the environment during the production, storage, transportation and use of CPs (POPRC, 2015). It was reported that the total emission of SCCPs in China reached 1789 tons in 2011 and will increase to 2563 tons in 2016 in the absence of any measures to reduce SCCP emission, with applications in metalworking fluids, flame retardants and plasticizer being the major emission sources (Xu et al., 2014).

Soils are a principal receptor and environmental reservoir for many persistent semi-volatile organic contaminants (SVOC) (Ockenden et al., 2003; Li et al., 2006; Gevao et al., 2011; Wang et al., 2012). Similar to other POPs, SCCPs can also accumulate in soils (Zeng et al., 2011). However, the current limitations of analytical methods make scarce information on SCCP pollution and their evolution in soils (Nicholls et al., 2001; Zeng et al., 2011; Gao et al., 2012; Wang et al., 2013ab, 2014; Halse et al., 2015). A study conducted in the Liaohe River Basin, where CPs were industrially produced in large quantities, found that local industrial activity was the major source of soil SCCP contamination (Gao et al., 2012). Similar results were observed for SCCPs in soils from the Pearl River Delta area (Wang et al., 2013b). In addition to emissions from local industrial sources, releases from sewage treatment plants, sludge application and wastewater irrigation were also suggested to be important sources of SCCPs in soils (Zeng et al., 2011; Wang et al., 2014).

Guangzhou is the largest city in southern China, whereas Chengdu is the largest city in western China (Fig. 1). As the capital of Guangdong Province, Guangzhou is located in the Pearl River Delta (PRD), an area that is well known as an industrial base and manufacturing center. The gross product of Guangzhou reached 16,707 billion yuan in 2014, ranking third nationwide (Zeng, 
2015). Leading industries in Guangzhou include automobile, electronic and petrochemical. A large amount of CPs may have been used in these industries in China, as CP products are largely used as metal cutting fluids, flame retardants and plasticizers (Xu et al., 2014). Guangzhou is subjected to the subtropical maritime monsoon climate, with the southeast and southwest monsoons prevailing in spring and summer and the northeast monsoon prevailing in autumn and winter (Chen et al., 2013a). Chengdu is the capital of Sichuang Province and is the most economically developed city in western China, with electronic information, pharmaceutical, aircraft, and tobacco being the main industries. In 2014, its gross product was 10,057 billion yuan (Yin, 2015). Chengdu is subjected to the subtropical humid monsoon climate. Although Chengdu is located in the monsoon region, the wind in Chengdu remains gentle throughout the year (CBS, 2011).

The different industrial structures between Guangzhou and Chengdu may lead to differences in the usage of CP products and cause further discrepancies in the status of SCCP pollution in the soils. In addition, the different climates of these two cities may also affect the soil SCCP levels (Wang et al., 2014). The primary objective of this study was to investigate the SCCP pollution status and composition profiles in urban and suburban soils from land of different use types in Guangzhou and Chengdu. Based on the surveyed data, the potential sources and pathways of SCCPs to the soils were further explored. Additionally, the vertical migration of SCCPs along soil columns and the role of organic matter $(\mathrm{OM})$ to the accumulation of SCCPs in soils were examined.

\section{Material and methods}

\subsection{Soil sampling design and collection}

Woodlands and vegetable and paddy fields are representative types of soils in urban and suburban areas. Vegetable and paddy fields are subjected to more anthropogenic disturbances than woodlands, including fertilization, irrigation and plowing. In the present study, 14 woodland topsoils (at a depth of 0-20 cm) (from no. 1 to no. 14), 22 vegetable field topsoils (from no. 15 to no. 36) and 8 paddy field topsoils (from no. 37 to no. 44) were collected from Guangzhou in July, 2009 (Fig. 1). Additionally, 6 woodland topsoils (from no. 45 to no. 50), 21 vegetable field topsoils (from no. 51 to no. 71) and 3 paddy field topsoils (from no. 72 to no. 74) were collected from Chengdu in November, 2010 (Fig. 1). Greenbelts are another important type of soil in urban areas and may be polluted by SCCPs from the application of sewage sludge and reclaimed wastewater irrigation (Wang et al., 2014). For this reason, 14 greenbelt topsoils (from no. 75 to no. 88) were collected during the sampling campaign in Chengdu (Fig. 1). To examine vertical migration of SCCPs in the soil, 10 soil columns were collected from the woodlands ( $\mathrm{n}=7$, nos. 1 , 2, 3, 4, 5, 10 and 14) and vegetable fields ( $\mathrm{n}=3$, nos. 25, 32 and 33) in Guangzhou, and 5 soil columns were collected from the woodlands $(\mathrm{n}=1$, no. 48$)$, vegetable fields $(\mathrm{n}=3$, nos. 59, 64 and 70) and paddy fields ( $\mathrm{n}=1$, no. 72) in Chengdu. Each soil column was separated into three soil samples: topsoil $(0-20 \mathrm{~cm})$, middle-layer soil $(20-40 \mathrm{~cm})$ and deep-layer soil $(40-60 \mathrm{~cm})$. 
Soil samples were wrapped in alumina foil, placed in polyethylene zip bags and then stored at $-20{ }^{\circ} \mathrm{C}$ until analysis. The alumina foils were baked at $450{ }^{\circ} \mathrm{C}$ for $4 \mathrm{~h}$ before use.

\subsection{Sample preparation and cleanup}

All soil samples were first dried for $24 \mathrm{~h}$ in a freeze-drier (FDU-2100, EYELA, Japan) and then ground and sifted through a 100-mesh stainless steel sieve. The soils were then Soxhlet extracted and purified according to our previously developed method (Chen et al., 2013b). Briefly, an aliquot of an approximate $20 \mathrm{~g}$ soil sample with $10 \mathrm{ng}$ of ${ }^{13} \mathrm{C}_{10}-\delta$-BHC added as the surrogate recovery was extracted using $200 \mathrm{~mL}$ DCM for $48 \mathrm{~h}$. To remove the sulfur that is potentially present in the soil, approximately $2 \mathrm{~g}$ of copper granule was added in the flask before extraction. The extract was concentrated and then cleaned on a silica gel column and a multilayer silica gel-florisil composite column in sequence. The purified SCCP fraction was concentrated to approximately $50 \mu \mathrm{L}$ under a gentle stream of nitrogen. Prior to instrumental analysis, $5 \mathrm{ng}$ of ${ }^{13} \mathrm{C}_{6}-\mathrm{HCB}$ was added as an internal standard.

The content of $\mathrm{OM}$ was determined using the $550^{\circ} \mathrm{C}$ loss-on-ignition method (Qian et al., 2011).

\subsection{Instrumental analysis}

SCCP concentrations were determined with an Agilent 6890 GC coupled with a 5975 MS in the electron capture negative ion mode (ECNI) (Santa Clara, USA) in the selective ion monitoring (SIM) mode. The separation was conducted in a DB-5 MS capillary column $(30 \mathrm{~m} \times 0.25 \mathrm{~mm}$, $0.25 \mu \mathrm{m}, \mathrm{J} \& \mathrm{~W}$ Scientific) with methane as the reagent gas. A $1 \mu \mathrm{L}$ aliquot of the sample was injected into the GC-MS system in the splitless mode. More information about the instrumental temperature procedure is detailed in the Supporting Information (SI). The scanning ions of the SCCP homologues were previously elucidated by Tomy et al. (1997). The scanning ions of

${ }^{13} \mathrm{C}_{10}-\delta$-BHC and ${ }^{13} \mathrm{C}_{6}$-HCB were $\mathrm{m} / z, 261$ and $\mathrm{m} / z$ 290, respectively. SCCPs were quantified using the method developed by Reth et al. (2005). The total relative response factors and chlorine contents of the nine SCCP standards were calculated to obtain the linear regression equation used in the present work. Piecewise linear regression equations were obtained from the total relative response factors, and the calculated chlorine contents of the nine SCCP standards with SCCP of $62.1 \%$ (chlorine content) were identified as the breakpoint.

\subsection{Quality assurance and quality control ( $Q A / Q C)$}

SCCP standards with 55.5\% and $62.1 \%$ chlorine content were injected every 20 samples to verify the validity of the standard curves. New curves were generated if the recovery of the injected SCCP standard were out of the range $80-120 \%$. The instrumental detection limit (IDL) was $250 \mathrm{pg}, 100 \mathrm{pg}$ and $30 \mathrm{pg}$ for SCCPs with chlorine content of 51.5\%, 55.5\% and 63\%, respectively. Spiked matrix samples (20 ng 55.5\% SCCPs), spiked laboratory blanks (20 ng 55.5\% 
SCCPs), laboratory blanks and replicate samples were also performed in the QA/QC procedure. One laboratory blank was prepared for each six soil samples and one replicate was analyzed for each ten samples. The recoveries of SCCPs in the spiked samples were 116-131\% (Table S1). SCCP contents in the laboratory blanks were below the IDL. Relative standard deviation (RSD) values of replicates were $<10 \%$. Surrogate recoveries of ${ }^{13} \mathrm{C}_{10}-\delta$-BHC in the samples ranged from $77 \%$ to $126 \%$. The SCCP concentrations reported herein were not recovery corrected. In addition, our laboratory participated in the "First Round Inter-laboratory Study on the Analysis of Chlorinated Paraffins in Environmental Matrices" organized by the Institute for Environmental Studies (IVM) of the VU University in Amsterdam, the Netherlands in 2011. The results of the SCCP concentrations submitted by us were satisfactory and agreed well with the target values.

\subsection{Statistical analysis}

All statistical analyses were performed using SPSS v 13.0 for windows (SPSS Inc., Chicago, USA). Correlations were tested using Pearson correlation coefficient. SCCP concentrations in the topsoils were compared using independent sample t-test, whereas SCCP concentrations in different layers of the soil columns were compared using paired-samples t-test. The results of the statistical tests were considered statistically significant when $p<0.05$.

\section{Results and discussions}

\subsection{SCCP concentrations in the topsoils of two different cities}

Table 1 summarizes the SCCP concentrations obtained from the topsoils from the different sources in Guangzhou and Chengdu. SCCPs were detected in all samples from the two cities (Table S2). Significantly high values were observed in topsoil samples 26 and 34 from the vegetable fields in Guangzhou, with SCCP concentration of $372 \mathrm{ng} \mathrm{g}^{-1} \mathrm{dw}$ and $185 \mathrm{ng} \mathrm{g}^{-1} \mathrm{dw}$, respectively. Fertilization and water-irrigation may be the potential sources for the high SCCP levels in these two samples. In addition, it is important to note that these two samples were both collected from vegetable fields near the road. CPs are widely used as additive flame retardant in rubber (POPRC, 2015). SCCPs in the rubber-made automobile tires could enter the road dust and contribute to the high levels of SCCPs in the soils of nearby vegetable fields. SCCPs could also enter the ambient environments during the transportation of CP products (POPRC, 2015). Apart from those two samples, the SCCP concentrations in the topsoils from Guangzhou ranged from 1.45 to $25.5 \mathrm{ng} \mathrm{g}^{-1} \mathrm{dw}$, with an average value of $10.3 \mathrm{ng} \mathrm{g}^{-1} \mathrm{dw}$. The SCCP concentrations in the topsoils from Chengdu ranged from 0.218 to $3.26 \mathrm{ng} \mathrm{g}^{-1} \mathrm{dw}$, with an average of $1.43 \mathrm{ng} \mathrm{g}^{-1} \mathrm{dw}$. It should be noted that, the topsoil samples from Chengdu were collected about one year later than those from Guangzhou, indicating that topsoil samples from Chengdu may have received more CP pollution than those from Guangzhou. However, we observed that the SCCP concentrations in topsoils from Chengdu were much lower than the values reported for topsoils from Guangzhou in 
this study $(p<0.001)$. This significant difference of SCCP concentration may be related to emission sources. It was reported that the application of CPs in metalworking fluids, flame retardants and plasticizers were the major emission sources of SCCPs and that the increasing demand of CPs as flame retardants in China were mainly caused by applications in the automobile and electronic industries (Xu et al., 2014). We note that the automobile and electronic industries are the principal industries in Guangzhou (GBS, 2010), whereas the electronic information, pharmaceutical, aircraft, and tobacco industries are the leading industries in Chengdu (CBS, 2011). This indicates that the leading industries in Guangzhou could emit more SCCPs than those in Chengdu. Additionally, a previous study reported that the production capacity of CPs in Guangzhou is much higher than in Chengdu (Tang and Yao, 2005). SCCPs are also released during the production, storage and transport of CPs (POPRC, 2015). Climatic conditions may be another factor influencing the SCCP concentration in topsoils. Affected by the oceanic monsoons, the average annual precipitation in Guangzhou (ca. $1800 \mathrm{~mm}$ ) (GBS, 2010) is much higher than that in Chengdu (ca. $900 \mathrm{~mm}$ ) (CBS, 2011). Precipitation is an important deposition pathway for the transfer of SVOC from the atmosphere to soil (Liu et al., 2014). Although the precipitation fluxes of SCCPs have not been investigated in these two cities, the relatively high precipitation in Guangzhou is considered to be more efficient for scavenging SCCPs from the atmosphere to soil.

SCCP concentrations in the topsoils of Guangzhou and Chengdu were compared with those reported for other areas (Table 2). Generally, SCCP concentrations in the topsoils from Guangzhou and Chengdu were lower than the values reported for topsoils from Shanghai (range: n.d.-615 ng $\mathrm{g}^{-1} \mathrm{dw}$, average: $39.4 \mathrm{ng} \mathrm{g}^{-1} \mathrm{dw}$ ) (Wang et al., 2014), the eastern PRD (range: 1.9-236 $\mathrm{ng} \mathrm{g}^{-1} \mathrm{dw}$, average: $18.3 \mathrm{ng} \mathrm{g}^{-1} \mathrm{dw}$ ) (Wang et al., 2013b), and the Liaohe River Basin (56.9-189.3 $\mathrm{ng} \mathrm{g}^{-1} \mathrm{dw}$ ) (Gao et al., 2012), and certainly lower than those reported for topsoils from seriously polluted areas such as sewage-irrigated farmland soils and e-waste polluted areas (Zeng et al., 2011; Chen et al., 2014) (Table 2).

\subsection{SCCP levels in topsoils from land of different use types}

With the exception of the significantly high SCCP concentrations in samples 26 and 34 (Table S2), the SCCP concentrations in the topsoils from the vegetable fields from Guangzhou ranged from 1.45 to $25.5 \mathrm{ng} \mathrm{g}^{-1}$, with an average value of $9.73 \pm 7.12 \mathrm{ng} \mathrm{g}^{-1}$, which was comparable to the values obtained for the woodland soils (average: $10.4 \pm 5.97 \mathrm{ng} \mathrm{g}^{-1}$ ) and paddy fields (average: $11.8 \pm 6.92 \mathrm{ng} \mathrm{g}^{-1}$ ) (Table 1). A similar distribution was observed for the topsoil samples from Chengdu. The SCCP concentrations in the topsoils from the woodland (average: $\left.1.05 \pm 0.552 \mathrm{ng} \mathrm{g}^{-1}\right)$ were comparable with soils from in the vegetable field $\left(1.16 \pm 0.559 \mathrm{ng} \mathrm{g}^{-1}\right)$ and paddy field $\left(1.30 \pm 0.217 \mathrm{ng} \mathrm{g}^{-1}\right)$ (Table 1). Although the vegetable and paddy fields have suffered more disturbances than the woodlands, such as fertilization, irrigation and plowing, there are no significant differences in the SCCP concentrations in the topsoils from these three types of land in either Guangzhou or Chengdu, with $p$ values $>0.05$. This may suggest that SCCPs in the 
topsoils of woodland, vegetable and paddy fields in Guangzhou and Chengdu are primarily influenced by atmospheric transport from industrial activities. Gas exchange and atmospheric deposition were suggested to be the main pathways of SCCP pollution in the soil of this region (Wang et al., 2013b). The SCCP concentrations in the topsoils from the greenbelt in Chengdu were $2.02 \pm 0.537 \mathrm{ng} \mathrm{g}^{-1}$, which are significantly higher than the topsoils in the woodland $(p<0.01)$, vegetable field $(p<0.01)$, and paddy fields $(p<0.05)$ in Chengdu. These results indicate that, in addition to atmospheric transport, there are other potential sources of SCCPs in the greenbelt, such as the use of sewage sludge and wastewater irrigation (Wang et al., 2014). Reclaimed wastewater and sewage sludge from sewage treatment plants could also be an important source of SCCPs to the ambient environment (Stevens et al., 2003; Zeng et al., 2011, 2012). Furthermore, the contribution from road dust discussed above could also be a potential source of SCCPs to the greenbelt topsoils because the greenbelts are located near roads.

\subsection{SCCP homologue patterns in the topsoils}

The average carbon and chlorine homologue group profiles of SCCPs in the different types of soils from Guangzhou and Chengdu have been determined. Because SCCPs with less than 5 chlorine atoms could not be well detected with ECNI-MS, the homologue group profiles that were analyzed are limited to $\mathrm{C}_{10}-\mathrm{C}_{13}$ SCCPs with 5-10 chlorine atoms. As shown in Fig. 2 and Fig. 3, $\mathrm{C}_{10}$ and $\mathrm{C}_{11}$ SCCPs were, in general, the major homologue groups, with $\mathrm{Cl}_{6-10}$ being the main group for $\mathrm{C}_{10} \mathrm{SCCP}$ and $\mathrm{Cl}_{6-8}$ being the main group for $\mathrm{C}_{11}, \mathrm{C}_{12}$ and $\mathrm{C}_{13}$ SCCPs in the soils from Guangzhou. The abundance of $\mathrm{Cl}_{10}$ decreased as the number of carbon atoms increased. The average homologue patterns of SCCPs in the soils from the paddy field, woodland and vegetable field of Chengdu were similar to those detected for Guangzhou, with $\mathrm{C}_{10} \mathrm{Cl}_{6-10}$ and $\mathrm{C}_{11} \mathrm{Cl}_{6-8}$ being the predominant homologue groups. For topsoils in the greenbelt, the average percentage of $\mathrm{C}_{10}$ (25.1\%) was comparable to those of $\mathrm{C}_{11}(25.4 \%), \mathrm{C}_{12}(23.4 \%)$ and $\mathrm{C}_{13}(26.2 \%)$, with $\mathrm{Cl}_{9-10}$ SCCPs being the main group of $\mathrm{C}_{10}$ SCCPs and $\mathrm{Cl}_{7-9}$ SCCPs being the main group of $\mathrm{C}_{11}, \mathrm{C}_{12}$, and $\mathrm{C}_{13}$ SCCPs. The average percentages of $\mathrm{C}_{12}$ and $\mathrm{C}_{13}$ in the greenbelt were relatively higher than those for topsoils from the woodland and vegetable and paddy fields (Fig. 3). These results indicate that the greenbelt soils are likely to be closer to SCCP emission sources, such as the road dust noted above, as heavier SCCPs have lower mobility than lighter SCCPs (Wang et al., 2013b). Moreover, the use of sewage sludge may also cause higher percentage of heavier SCCPs in the greenbelt topsoils, as $\mathrm{C}_{12}$ and $\mathrm{C}_{13}$ are more capable of enriching in sewage sludge (Zeng et al., 2013).

In the present work, the SCCP patterns in the topsoils from Guangzhou and Chengdu are different from those in the urban soils of Shanghai, where the most abundant homologue groups were $\mathrm{C}_{11} \mathrm{Cl}_{5-7}$ and $\mathrm{C}_{13} \mathrm{Cl}_{5-7}$ (Wang et al., 2014). Compared with the results for the soils from the paddy fields and uplands in the Liaohe River Basin (Gao et al., 2012), the abundances of the $\mathrm{C}_{10}$ and $\mathrm{C}_{11}$ homologues were relatively low in the present study. Meanwhile, the homologue patterns of SCCPs differ from those of the CP mixtures reported by Chen et al. (2011) and Gao et al. 
(2012). However, the main source of SCCPs in the topsoils from Guangzhou and Chengdu is suggested to be CP-52 because it contributed almost $90 \%$ of the total CP output in China (WCC, 2014) and because it has much higher fractions of SCCPs than CP-42 and CP-70 (Gao et al., 2012). Further, the homologue patterns of SCCPs in the present work are neither similar to those of the CP-52 product analyzed by Chen et al. (2011) nor similar to those analyzed by Gao et al. (2012). This may occur because of differences in raw materials and technologies in the production of CP-52 in China (Tang and Yao, 2005; Xu et al., 2014). In addition, the complex environmental processes (e.g., partition, dechlorination, fractionation, and degradation) that the SCCPs may have experienced could also affect the homologue patterns of SCCPs in the topsoils analyzed in the present work (Wang et al., 2013b).

\subsection{The relationship between $\mathrm{OM}$ and SCCP concentrations}

In addition to being influenced by industrial sources, the concentrations of organic pollutants in the soil may be influenced by the content of OM in the soil (Wang et al., 2012). Previous studies have suggested that the presence of $\mathrm{OM}$ in soils played an important role in controlling the sorption of POPs (e.g., DDTs, PCBs) (Ribes et al., 2002; Gong et al., 2004; Chen et al., 2008; Liu et al., 2014). Zeng et al. (2011) reported that SCCP concentrations in the soil cores of sewage-irrigated farmland were significantly correlated with the total organic carbon (TOC) $\left(\mathrm{R}^{2}\right.$ : $0.32-0.85, p<0.05$ ). A significant positive correlation was also observed between the TOC and CP concentrations in sediments of the Pearl River Delta (Chen et al., 2011). However, in other studies, the SCCP concentrations in the soil were found to possess no significant relationship with TOC (Gao et al., 2012; Wang et al., 2013a; Wang et al., 2013b; Chen et al., 2014; Wang et al., 2014). For a more direct estimate of the influence of OM, the OM content in the soils was determined in this study (Table 1). We examined the Pearson correlations among OM values and SCCP concentrations for the different types of topsoils. The results suggest that the SCCP concentrations in the topsoils from the vegetable fields in Chengdu were significantly related to the OM levels $(R=0.478, p=0.029)$, whereas the SCCP concentrations in the other types of topsoils from Guangzhou and Chengdu displayed little dependence on the OM levels (Table 1).

\subsection{Vertical migration of SCCPs along soil columns}

The SCCP concentrations in the top $(0-20 \mathrm{~cm})$, middle-layer $(20-40 \mathrm{~cm})$, and deep-layer $(40-60 \mathrm{~cm})$ of soil columns were analyzed to investigate the vertical variation of SCCPs.

As shown in Fig. 4, for most of the soil columns from the woodland, the SCCP concentrations hardly varied among the topsoil, middle-layer soil and deep-layer soils, with $p$ values $>0.05$. Because the woodland soils are seldom affected by human activities, SCCPs in the "aged" middle- and deep-layers may be related to a range of processes such as water driven leaching (Zeng et al., 2011), vertical diffusion and bioturbation. These processes drive the downward vertical transport of SCCPs in the soil column, as suggested for flame retardants in 
forest soils (Zheng et al., 2015). The composition profiles of SCCPs in the woodland soil columns suggest that the proportion of $\mathrm{Cl}_{5-6}$ increased from the topsoils to the deep-layer soils, whereas the proportion of $\mathrm{Cl}_{9-10}$ decreased with increased depth (Fig. 5). In the soil column from sampling site 1, the SCCP concentration in the deep-layer soil was four times higher than that in the topsoil and middle-layer soil. Meanwhile, the vertical variation of the SCCP pattern along the soil column from sampling site 1 was larger than the other soil columns, with the relative abundance of $\mathrm{Cl}_{5-6}$ and $\mathrm{Cl}_{9-10}$ in the topsoil being $10.7 \%$ and $48.1 \%$, respectively, changing to $32.4 \%$ and $12.5 \%$, respectively, in the deep-layer soil (Fig. 5). These results indicated that the less chlorinated SCCPs in the topsoils of woodland columns could be more likely to reach the deep-layer soils to some extent because of water driven leaching. It was found that SCCPs with less chlorines and carbons had lower comparatively values than SCCPs with more chlorines and carbons (Sijm and Sinnige, 1995). Higher water solubility of the less chlorinated SCCPs would enhance their potential to leach downward the soil column (Zeng et al., 2011). What's more, it was also possible that $\mathrm{Cl}_{10}$ and $\mathrm{Cl}_{9} \mathrm{SCCPs}$ dechlorine to less chlorinated SCCPs in the soil columns.

SCCP concentrations in the topsoils of vegetable and paddy field soil columns were found to be higher than those in the middle- and deep-layer samples (Fig. 4). Similar to the vertical variation in the woodland soil columns, the abundance of $\mathrm{Cl}_{5-6}$ in the vegetable and paddy field soil columns increased from the topsoils to deep-layer soils with the $\mathrm{Cl}_{9-10}$ proportion decreasing with increased depth (Fig. 5). This may be related to mobility differences between the different SCCP homologues, as discussed above. Furthermore, water irrigation accelerates the vertical migration of SCCPs via water leaching, in particular for the less chlorinated SCCPs, and especially in the soil columns from sampling sites 64, 70 and 72 (Fig. 5). Similar observations were found in the soil cores from wastewater-irrigated farmlands in Beijing suburbs (Zeng et al., 2011) and in a sediment core from the Dongjiang River (Chen et al., 2011).

\section{Conclusions}

The present work analyzed the SCCP concentrations and homologue patterns in soils from Guangzhou and Chengdu. The SCCP concentrations in the topsoils from Guangzhou and Chengdu were quite low relative to the values reported for topsoils from other areas. Much higher SCCP concentrations were observed in Guangzhou than in Chengdu, which may be related to the differences in the SCCP emissions and climates of these two cities. No significant differences were observed for the SCCP concentrations in the topsoils from woodlands and vegetable or paddy fields in either Guangzhou or Chengdu, indicating that atmospheric transport from industrial activities was their common source and that gas exchange and atmospheric deposition were the main pathways. Pearson correlation analysis for SCCPs and OM indicated that OM is not the leading factor influencing the soil SCCP concentrations. Vertical migration of SCCPs is a complex process. The vertical variations of the SCCP homologue pattern along the soil columns suggested that less chlorinated SCCPs with smaller $K_{\mathrm{ow}}$ values may be more prone to move to the 
deep-layer soils under the role of water-driven leaching. More attention should be given to SCCP pollution in the greenbelt from sewage sludge application and sewage irrigation. Additionally, because two significantly high SCCP concentrations have been observed in the two topsoils from vegetable field soils in Guangzhou with uncertain sources, follow-up investigations should be performed to examine SCCP pollution in vegetable field soils and their associated vegetables. Finally, SCCPs pollution caused by road dust also requires further investigation.

Acknowledgments: This study was funded by the Commonwealth and Environmental Protection Project of the Ministry of Environmental Protection (MEP) of the People's Republic of China (No.200909090), the National Science Foundation of China (No. 41273107 and 41573123) and the Emerging Artist Scheme of Guangzhou University (No. 2015-08).

\section{References:}

Bayen S, Obbard JP, Thomsa GO. Chlorinated paraffins: A review of analysis and environmental occurrence. Environ Int 2006; 32: 915-29.

CBS (Chengdu Bureau of Statistics). Chengdu Statistical Yearbook (2011). Beijing: China Statistics Press, 2011.

Chen D, Liu W, Liu X, Westgate JN, Wania F. Cold-trapping of persistent organic pollutants in the mountain soils of Western Sichuan, China. Environ Sci Technol 2008; 42: 9086-91.

Chen M-Y, Luo X-J, Zhang X-L, He M-J, Chen S-J, Mai B-X. Chlorinated paraffins in sediments from the Pearl River Delta, South China: Spatial and temporal distributions and implication for processes. Environ Sci Technol 2011; 45: 9936-43.

Chen L, Liu M, Xu Z, Fan R, Tao J, Chen D, et al. Variation trends and influencing factors of total gaseous mercury in the Pearl River Delta-A highly industrialised region in South China influenced by seasonal monsoons. Atmos Environ 2013a; 77: 757-66.

Chen L, Huang Y, Han S, Feng Y, Jiang G, Tang C, et al. Sample pretreatment optimization for the analysis of short chain chlorinated paraffins in soil with gas chromatography-electron capture negative ion-mass spectrometry. J Chromatogr A 2013b; 1274: 36-43.

Chen R, Wang YW, Wang P, Jiang GB. Spatial distribution of short chain chlorinated paraffins in soils from Taizhou, an e-waste dismantling area. Environ Chem 2014; 33(6): 873-9. [in Chinese].

Gao Y, Zhang H, Su F, Tian Y, Chen J. Environmental occurrence and distribution of short chain chlorinated paraffins in sediments and soils from the Liaohe River Basin, P. R. China. Environ Sci Technol 2012; 46: $3771-8$.

GBS (Guangzhou Bureau of Statistics). Guangzhou Statistical Yearbook (2010). Beijing: China Statistics Press, 2010.

Gevao B, Ghadban AN, Uddin S, Jaward FM, Bahloul M, Zafar J. Polybrominated diphenyl ethers (PBDEs) in soils along a rural-urban-rural transect: Sources, concentration gradients, and profiles. Environ Pollut 2011; 159 (12): $3666-72$

Gong ZM, Tao S, Xu FL, Dawson R, Liu WX, Cui YH, et al. Level and distribution of DDT in surface soils from Tianjin, China. Chemosphere 2004; 54: 1247-53.

Halse AK, Schlabach M, Schuster JK, Jones KC, Steinnes E, Breivik K. Endosulfan, pentachlorobenzene and short-chain chlorinated paraffins in background soils from Western Europe. Environ Pollut 2015; 196: 21-8. 
Li J, Zhang G, Qi S, Li X, Peng X. Concentrations, enantiomeric compositions, and sources of HCH, DDT and chlordane in soils from the Pearl River Delta, South China. Sci Total Environ 2006; 372: 215-24.

Liu X, Li J, Zheng Q, Bing H, Zhang R, Wang Y, et al. Forest filter effect versus cold trapping effect on the altitudinal distribution of PCBs: A case study of Mt. Gongga, eastern Tibetan Plateau. Environ Sci Technol 2014; 48: 14377-85.

Nicholls CR, Allchin CR, Law RJ. Level of short and medium chain length polychlorinated n-alkanes in environmental samples from selected industrial areas in England and Wales. Environ Pollut 2001; 114: 415-30.

Ockenden WA, Breivik K, Meijer SN, Steinnes E, Sweetman AJ, Jones KC. The global re-cycling of persistent organic pollutants is strongly retarded by soils. Environ Pollut 2003; 121: 75-80.

POPRC (Persistent Organic Pollutants Review Committee). Report of the Persistent Organic Pollutants Review Committee on the work of its eleventh meeting-Addendum: Risk profile on short-chained chlorinated paraffins. UNEP/POPS/POPRC.11/10/Add.2. Stockholm Convention on Persistent Organic Pollutants, 2015.

Qian B, Liu L, Xiao X. Comparative tests on different methods for content of soil organic matter. J Hohai Univ (Nat Sci) 2011; 39(1): 34-8. [in Chinese].

Reth M, Zencak Z, Oehme M. New quantification procedure for the analysis of chlorinated paraffins using electron capture negative ionization mass spectrometry. J Chromatogr A 2005; 1081: 225-31.

Ribes A, Grimalt JO, Torres García, CJ, Cuevas E. Temperature and organic matter dependence of the distribution of organochlorine compounds in mountain soils from the subtropical Atlantic (Teide, Tenerife Island). Environ Sci Technol 2002; 36: 1879-85.

Sijm D, Sinnige TL. Experimental octanol/water partition coefficients of chlorinated paraffins. Chemosphere 1995; 31 (11-12): 4427-35.

Stevens JL, Northcott GL, Stern GA, Tomy GT, Jones KC. PAHs, PCBs, PCNs, organochlorine pesticides, synthetic musks, and polychlorinated n-alkanes in UK sewage sludge: Survey results and implications. Environ Sci Technol 2003; 37 (3): 462-7.

Sun YJ, Wang XG, Li J, Yu J. Current situation and prospects of chlorinated paraffins. Chlor-Alkali Industry 2005; 6: 26-9. [in Chinese].

Tang ET, Yao LQ. Industry status of chlorinated paraffin and its development trends. China Chlor-Alkali. 2005, 2: 1-3. [in Chinese].

Tomy GT, Stern GA, Muir DCG, Fisk AT, Cymbalisty CD, Westmore JB. Quantifying $\mathrm{C}_{10}-\mathrm{C}_{13}$ polychloroalkanes in environmental samples by high-resolution gas chromatography/electron capture negative ion high-resolution mass spectrometry. Anal Chem 1997; 69(14): 2762-71.

Wang X-T, Zhang Y, Miao Y, Ma L-L, Li Y-C, Chang Y-Y, et al. Short-chain chlorinated paraffins (SCCPs) in surface soil from a background area in China: Occurrence, distribution, and congener profiles. Environ Sci Pollut Res 2013a; 20: 4742-9.

Wang X-T, Wang X-K, Zhang Y, Chen L, Sun Y-F, Li M, et al. Short- and medium-chain chlorinated paraffins in urban soils of Shanghai: Spatial distribution, homologue group patterns and ecological risk assessment. Sci Total Environ 2014; 490: 144-52.

Wang Y, Cheng Z, Li J, Luo C, Xu Y, Li Q, et al. Polychlorinated naphthalenes (PCNs) in the surface soils of the Pearl River Delta, South China: Distribution, sources, and air-soil exchange. Environ Pollut 2012; 170: 1-7.

Wang Y, Li J, Cheng Z, Li Q, Pan X, Zhang R, et al. Short- and medium-chain chlorinated paraffins in air and soil of subtropoical terrestrial environment in the Pearl River Delta, South China: Distribution, composition, atmospheric deposition fluxes, and environmental fate. Environ Sci Technol 2013b; 47: 2679-87.

WCC (World Chlorine Council). International Chlorinated Alkanes Industry Association (ICAIA) newsletter, 2013. <http://www.eurochlor.org/media/88255/20130712_icaia_newsletter_02_final.pdf.> 
WCC (World Chlorine Council). International Chlorinated Alkanes Industry Association (ICAIA) newsletter, 2014. <http://www.eurochlor.org/media/88258/20140908_icaia_newsletter_03_final.pdf.>

$\mathrm{Xu} \mathrm{C,} \mathrm{Xu} \mathrm{J,} \mathrm{Zhang} \mathrm{J.} \mathrm{Emission} \mathrm{inventory} \mathrm{prediction} \mathrm{of} \mathrm{short} \mathrm{chain} \mathrm{chlorinated} \mathrm{paraffins} \mathrm{(SCCPs)} \mathrm{in} \mathrm{China.} \mathrm{Acta}$ Sci Nat Univ Pekin 2014; 50(2): 369-78. [in Chinese].

Yin T. Chengdu's economic developed fast in 2014, with gross domestic product more than one trillion. Chengdu Daily 2015-01-29. [in Chinese]

Zeng N. The gross domestic product of Guangzhou ranking third nationwide. Nanfang Daily 2015-02-10. [in Chinese]

Zeng L, Wang T, Han W, Yuan B, Liu Q, Wang Y, et al. Spatial and vertical distribution of short chain chlorinated paraffins in soils from wastewater irrigated farmlands. Environ Sci Technol 2011; 45: 2100-6.

Zeng L,Wang T, Ruan T, Liu Q, Wang Y, Jiang G. Levels and distribution patterns of short chain chlorinated paraffins in sewage sludge of wastewater treatment plants in China. Environ Pollut 2012; 160: 88-94.

Zeng L, Li H, Wang T, Gao Y, Xiao K, Du Y, et al. Behavior, fate, and mass loading of short chain chlorinated paraffins in an advanced municipal sewage treatment plant. Environ Sci Technol 2013; 47: 732-40.

Zheng Q, Nizzetto L, Li J, Mulder MD, Sáňka O, Lammel G, et al. Spatial distribution of old and emerging flame retardants in Chinese forest soils: Sources, trends and processes. Environ Sci Technol 2015; 49: 2904-11. 


\section{Table 1}

Summary of SCCP concentrations (ng $\mathrm{g}^{-1} \mathrm{dw}$ ), chlorine contents (\%), OM levels ( $\mathrm{mg} \mathrm{g}^{-1} \mathrm{dw}$ ), and Pearson correlation coefficients between SCCP concentrations and OM levels in topsoils from land of different use types

\begin{tabular}{lccccc}
\hline \multicolumn{1}{c}{ Soil type } & Number & SCCPs & Chlorine content & OM & $\begin{array}{c}\text { Pearson correlation } \\
\text { coefficient }\end{array}$ \\
\hline Woodland & 14 & $2.96-23.6^{\mathrm{a}}(10.4)^{\mathrm{b}}$ & $61.4-65.4(63.6)$ & $41.1-209(96.0)$ & $0.093(p>0.05)$ \\
Vegetable field & 22 & $1.45-372(34.2)$ & $61.1-65.0(63.3)$ & $46.9-206(96.4)$ & $-0.102(p>0.05)$ \\
Paddy field & 8 & $2.97-23.1(11.8)$ & $62.0-64.7(63.1)$ & $72.2-292(130)$ & $0.156(p>0.05)$ \\
\hline & & & Chengdu & & \\
\hline Woodland & 6 & $0.321-1.74(1.05)$ & $61.0-64.6(63.1)$ & $45.4-91.4(69.0)$ & $0.038(p>0.05)$ \\
Vegetable field & 21 & $0.218-2.29(1.16)$ & $61.7-65.6(63.5)$ & $37.9-127(70.9)$ & $0.478(p<0.05)$ \\
Paddy field & 3 & $1.14-1.54(1.30)$ & $63.5-64.3(63.8)$ & $61.5-65.7(63.2)$ & $-0.465(p>0.05)$ \\
Greenbelt & 14 & $1.02-3.26(2.02)$ & $63.3-67.5(65.1)$ & $60.5-217(86.2)$ & $0.002(p>0.05)$ \\
\hline
\end{tabular}

${ }^{\mathrm{a}}$ concentration range; ${ }^{\mathrm{b}}$ the average value.

\section{Table 2}

SCCP concentrations ( $\mathrm{ng} \mathrm{g}^{-1} \mathrm{dw}$ ) in topsoils from different regions.

\begin{tabular}{|c|c|c|c|c|}
\hline Location & Soil type & $\begin{array}{l}\text { Sampling } \\
\text { time }\end{array}$ & SCCP concentrations & Reference \\
\hline Guangzhou $^{\mathrm{a}}$ & woodland, vegetable field, paddy field & 2009 & $1.45-25.5^{\mathrm{b}}(10.3)^{\mathrm{c}}$ & this study \\
\hline Chengdu & $\begin{array}{l}\text { woodland, vegetable field, paddy field, } \\
\text { greenbelt }\end{array}$ & 2010 & $0.218-3.26(1.43)$ & this study \\
\hline Shanghai & greenbelt, roadside, park, campus & 2011 & n.d. ${ }^{\mathrm{d}}-615(39.4)$ & Wang et al., 2014 \\
\hline Eastern Pearl River Delta & - & 2009 & $1.9-236(18.3)$ & Wang et al., 2013b \\
\hline Liaohe River Basin & paddy field, upland & 2010 & $56.9-189.3(114)$ & Gao et al., 2013 \\
\hline Southeast of Beijing & wastewater irrigated farmland & 2008-2009 & $159.9-1450$ & Zeng et al., 2011 \\
\hline Taizhou & e-waste polluted land & 2008-2009 & $47.95-1298(276.7)$ & Chen et al., 2014 \\
\hline
\end{tabular}

${ }^{\mathrm{a}}$ the two significantly high values in topsoils from vegetable field are excluded here; ${ }^{\mathrm{b}}$ concentration range; ${ }^{\mathrm{c}}$ the average value; ${ }^{\mathrm{d}}$ not detected. 


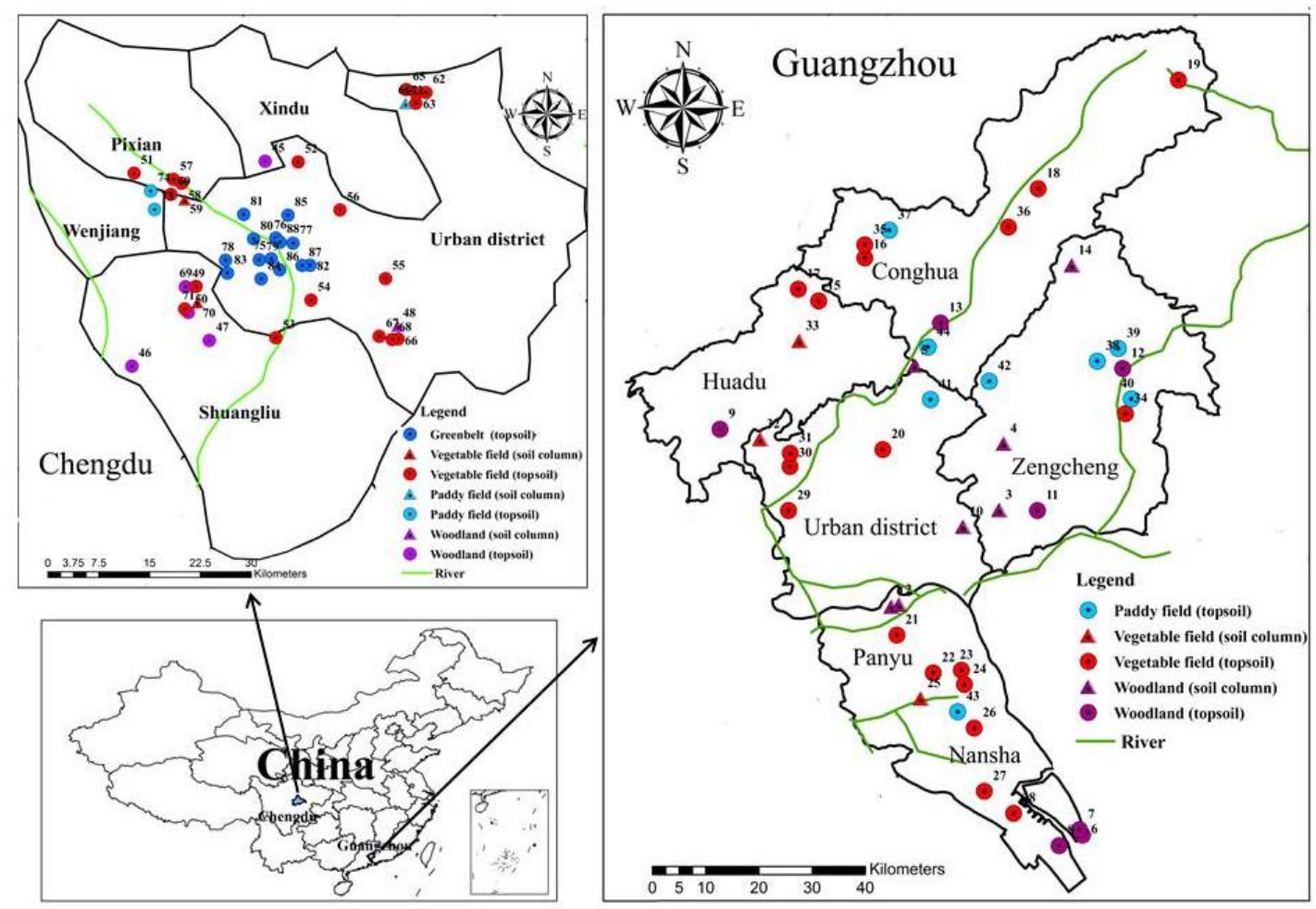

Fig. 1. Sampling sites of soils in Guangzhou and Chengdu.

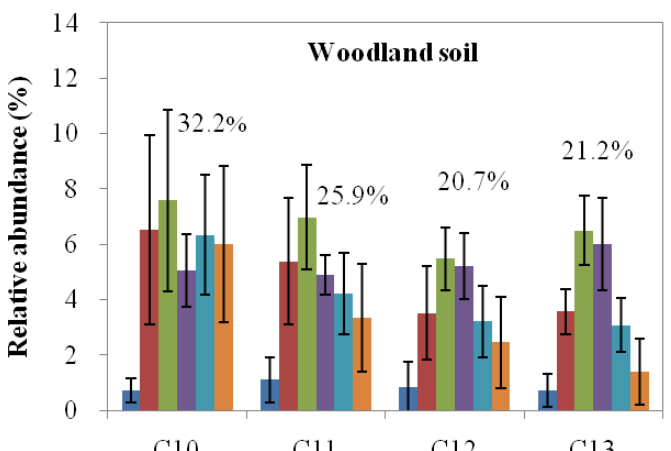

C10

C11

$\mathrm{C} 12$

$\mathrm{C} 13$
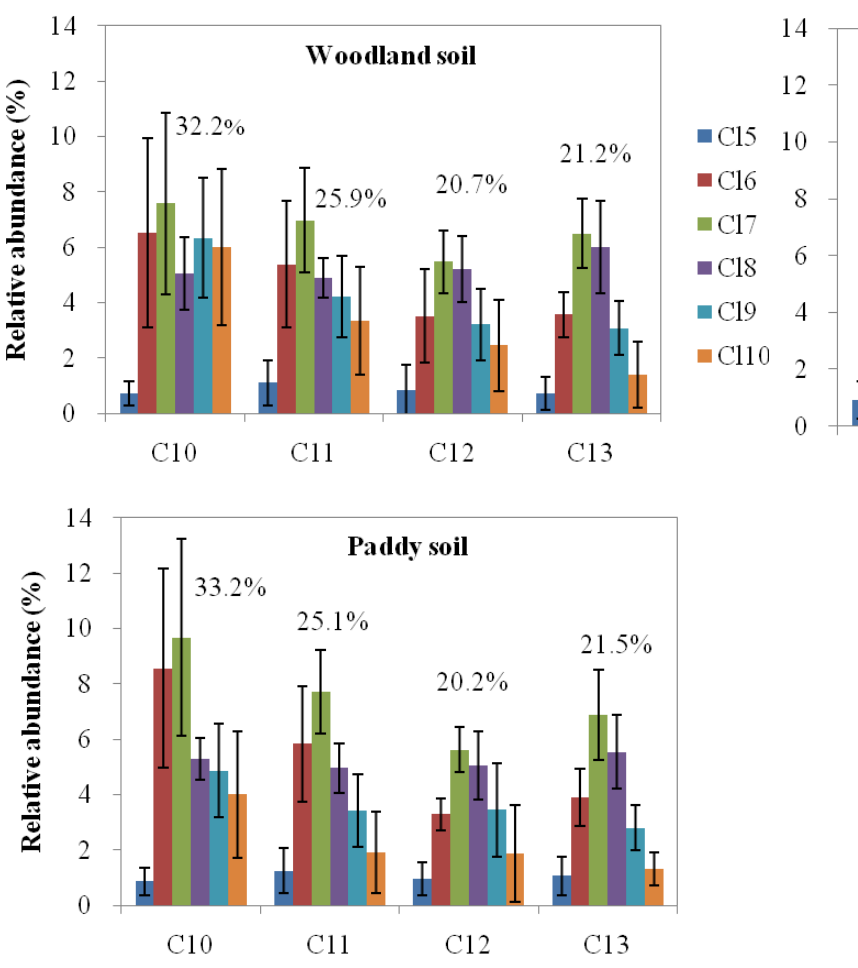

Fig. 2. Average homologue patterns of SCCPs in topsoils from land of different use types in Guangzhou. 

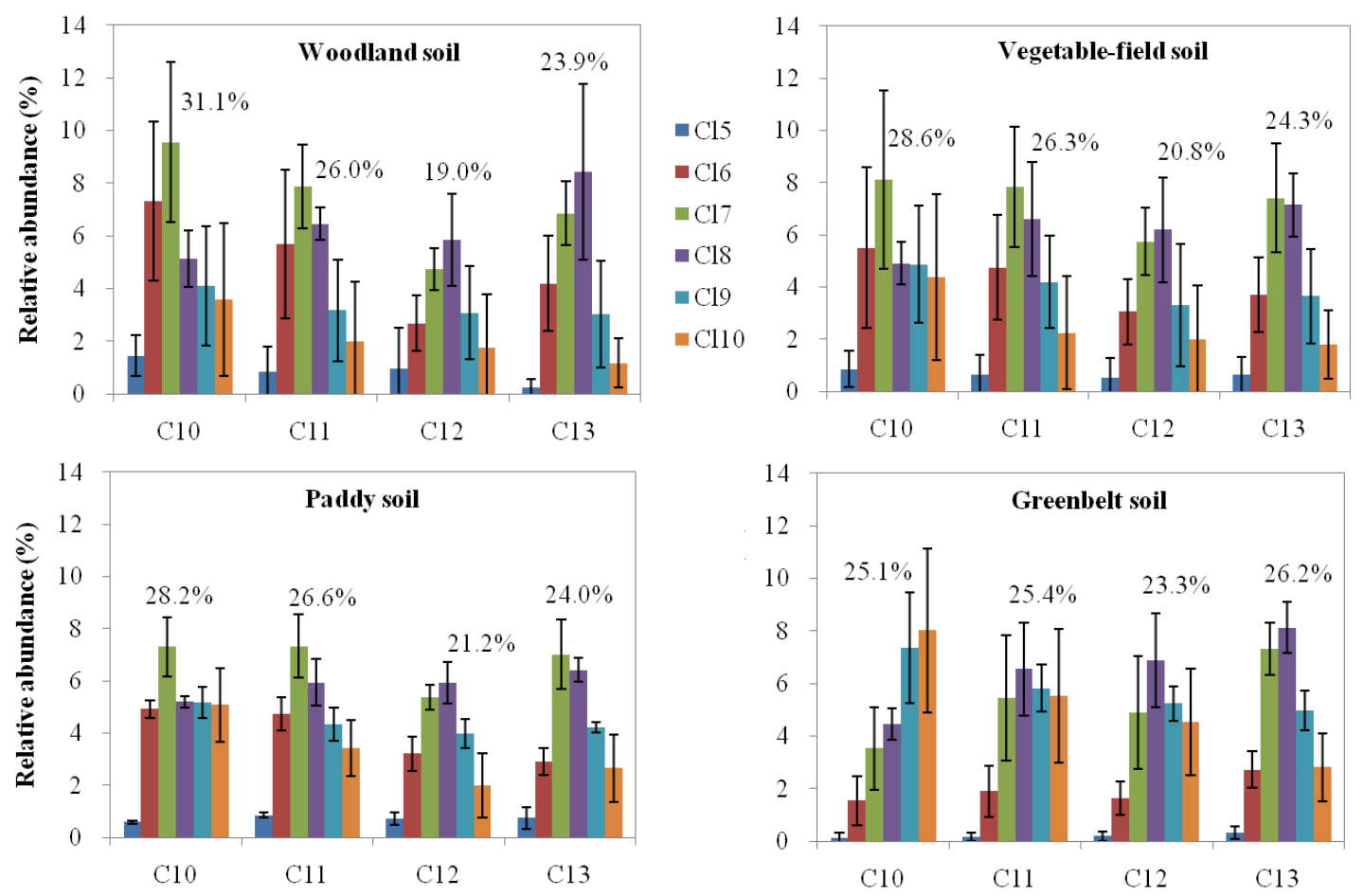

Fig. 3. Average homologue patterns of SCCPs in topsoils from land of different use types in Chengdu.

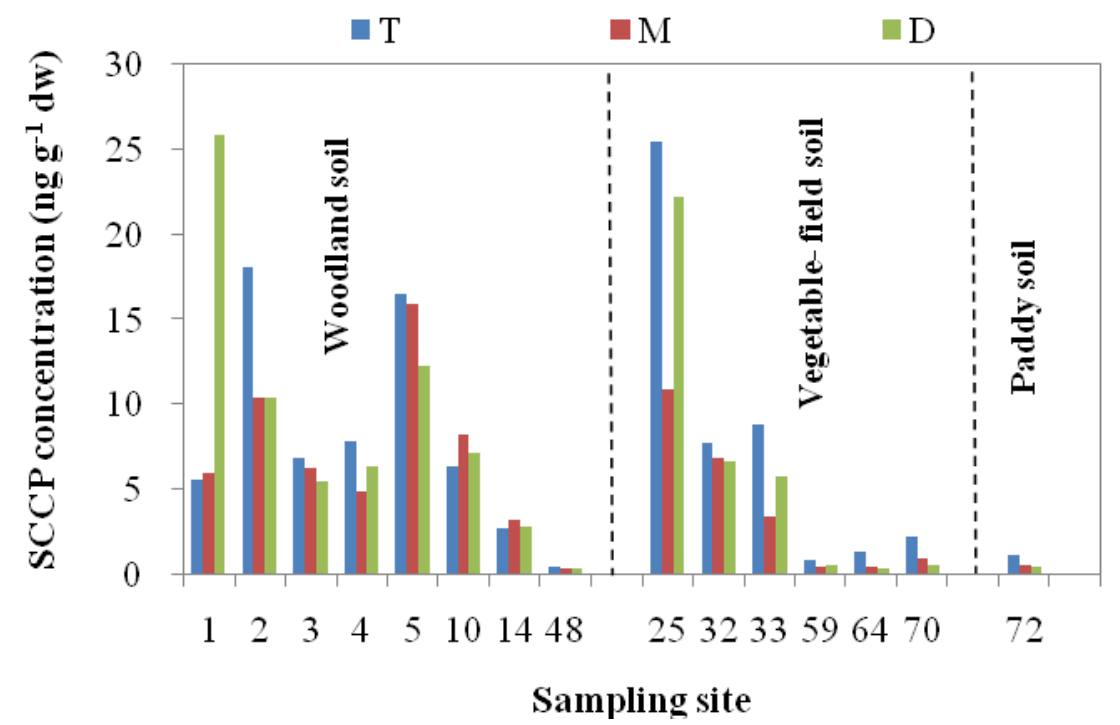

Fig.4. SCCP concentrations in topsoil (T), middle-layer soil (M) and deep-layer soil (D) of the soil columns from land of different use types 


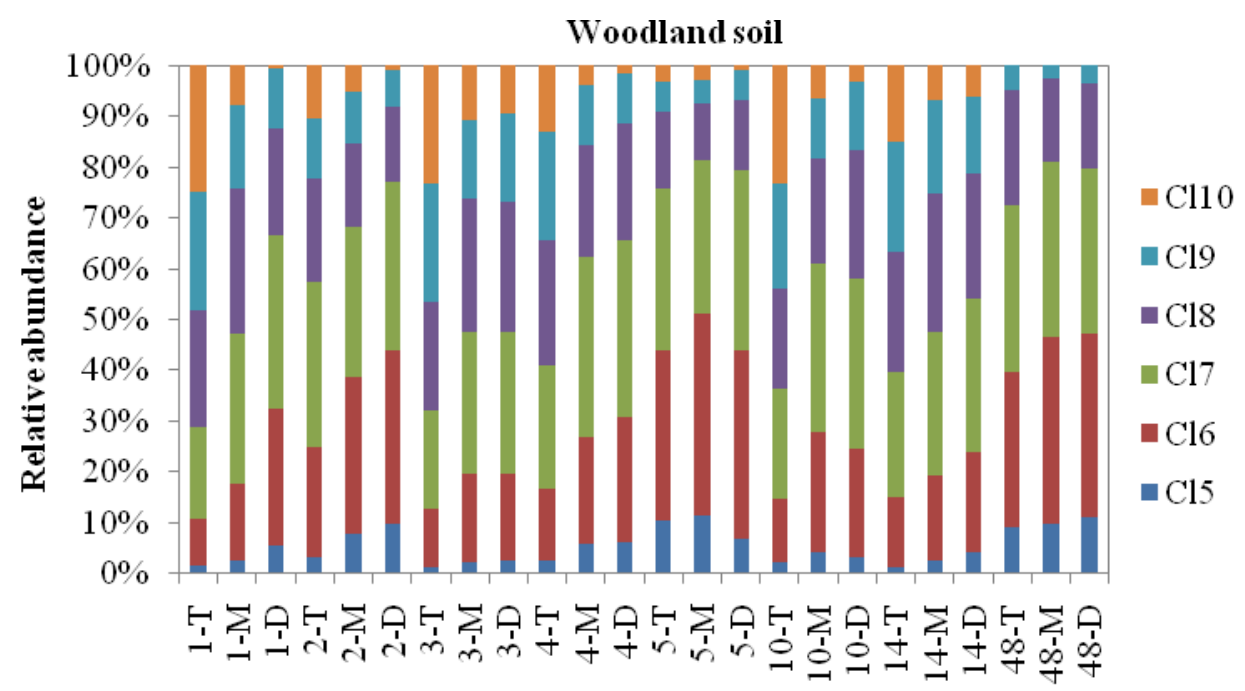

Sampling site

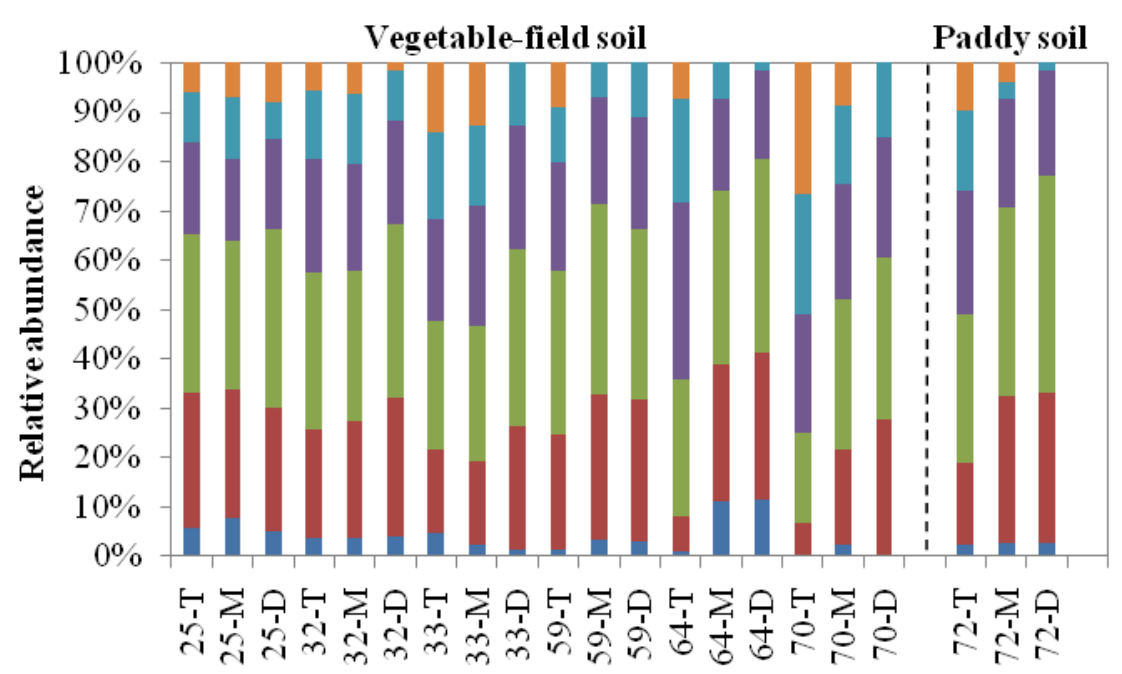

Sampling site

Fig. 5. Composition profiles of SCCP homologues with $\mathrm{Cl}_{5-10}$ for topsoil (T), middle-layer soil (M), and deep-layer soil (D) samples of the soil columns from land of different use types. 


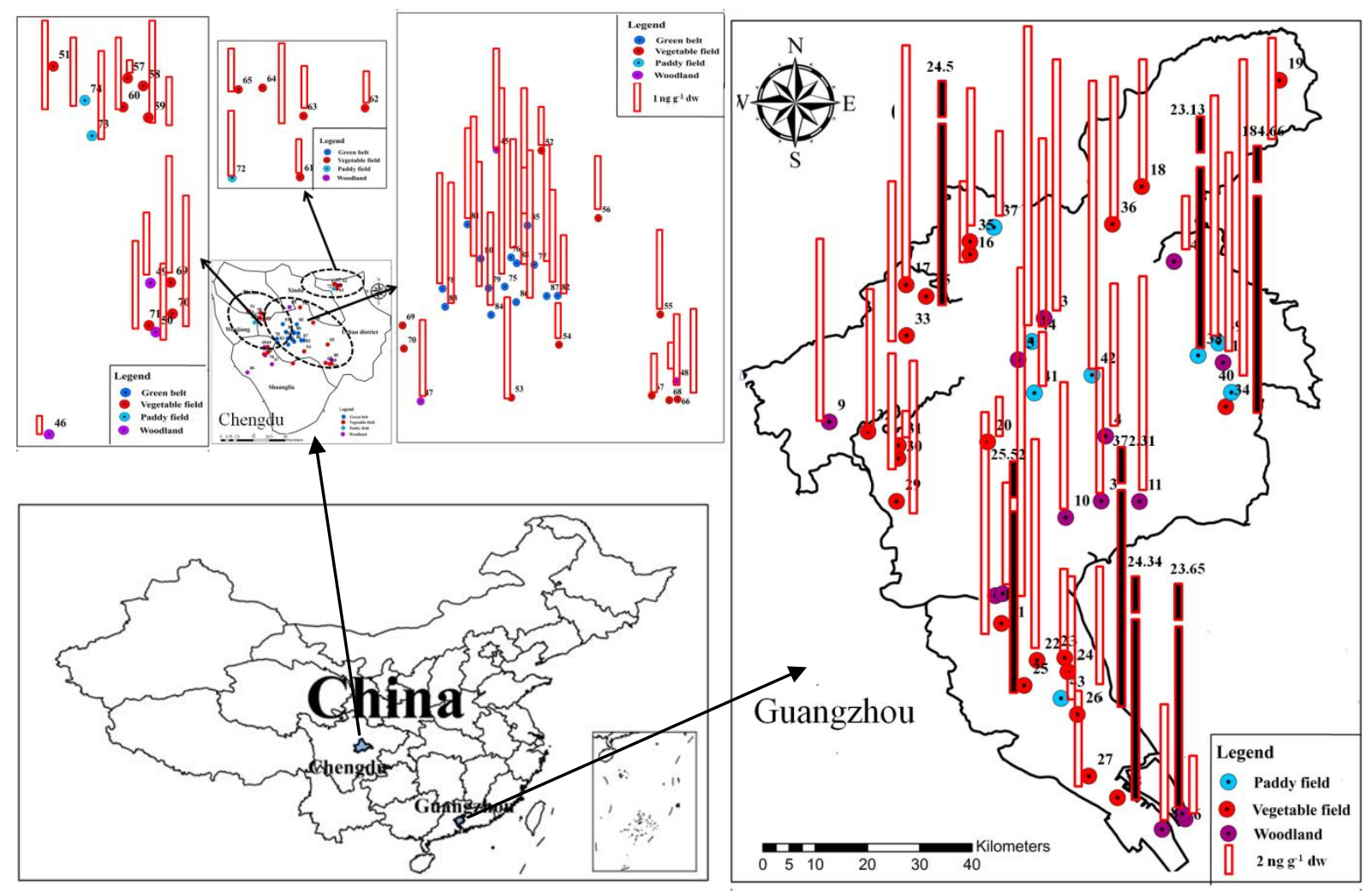

